\title{
Perfil sociodemográfico e epidemiológico de pacientes com AIDS residentes na região de saúde que compreende o município de Alagoinhas - BA, no período de 2007-2017
}

\author{
Sociodemographic and epidemiological profile of AIDS patients residing in the health \\ region comprising the Alagoinhas - BA county, period 2007-2017
}

\author{
Camilla de Souza Cerqueira ${ }^{1}$, Isabela Silva Pita Sereno ${ }^{1}$, Larissa Santana dos Santos Pinto ${ }^{1}$, Mayra \\ Mariana Lins de Azevedo Vila Verde ${ }^{1}$, Uilson Santos Pereira ${ }^{1}$, Carla Jaqueline Silva Sampaio ${ }^{2 *}$ \\ ${ }^{1}$ Graduanda em Medicina - Faculdade Estácio de Alagoinhas.; ${ }^{2}$ Doutora em Energia e Ambiente, Universidade \\ Federal da Bahia
}

\begin{abstract}
Resumo
Objetivo: este estudo teve como objetivo traçar o perfil sociodemográfico e epidemiológico de pacientes com AIDS residentes microrregião na região de saúde que compreende o município de Alagoinhas - BA, no período de 2007-2017. Metodologia: trata-se de um estudo epidemiológico descritivo, exploratório de fonte secundária de dados. As variáveis investigadas foram: gênero, faixa etária, grau de escolaridade, município de residência, cor, categoria de exposição e evolução do caso. Resultados: durante o período 2007- 2017 constataram-se 459 casos registrados no SINAN, de AIDS em adultos na Região de Saúde do município. Assim, verificou-se em adulto uma tendência de crescimento no número de casos na microrregião de Alagoinhas, acentuado no ano de 2014 (95 casos), e com considerável aumento constatado no ano de 2017 (90 casos). Evidenciou-se que 227 pacientes pertenciam ao sexo masculino e 232 ao sexo feminino, havendo predomínio de casos, todavia, na faixa etária entre 20 a 34 anos. Houve maior número de casos em adultos, faixa etária de 20-34 e 35-49, e dentre estes, o número de casos foi maior em indivíduos que têm relação heterossexual, seguido de indivíduos que têm relação homossexual; houve alto número de casos em indivíduos que possuem relações sexuais só com homens, totalizando 258, seguido de só com mulheres (135). Conclusão: o presente estudo permitiu compreender que os indivíduos adultos vivendo com HIV/AIDS, no período de 2007 a 2017, seguem a tendência nacional, contudo a melhoria do acesso aos serviços, qualidade da atenção, proporciona uma maior qualidade de vida aos pacientes.

Palavras-Chave: HIV/Aids. Epidemiologia. SINAN.
\end{abstract}

\begin{abstract}
Objective: this study aimed to outline the sociodemographic and epidemiological profile of AIDS patients living in - the health region of the Health Region comprising the municipality of Alagoinhas - BA, in the period 2007-2017. Methodology: this is a descriptive, exploratory epidemiological study of a secondary source of data. The variables investigated were: gender, age group, education level, municipality of residence, color, category of exposure and evolution of the case. Results: during the period 2007-2017, 459 cases were recorded in the SINAN of AIDS in adults in the Health Region of the municipality. Thus, in adults, there was a trend of growth in the number of cases in the Alagoinhas microregion, accentuated in 2014 (95 cases), and with a considerable increase observed in 2017 (90 cases), it was evidenced that 227 patients belonged were male, and 232 were female, with cases predominating, however, in the age group between 20 and 34 years. There were more cases in adults, age range 20-34 and 35-49, and among these, the number of cases was higher in individuals who have a heterosexual relationship, followed by individuals who have a homosexual relationship, there were a high number of cases in individuals who have sex with men alone, totaling 258, followed by only with women (135). Conclusion: the present study made it possible to understand that adult individuals living with HIV / AIDS, from 2007 to 2017, follow the national trend, however, improving access to services and quality of care provides a better quality of life for patients.

Keywords: HIV / Aids. Epidemiology. SINAN.
\end{abstract}

\section{INTRODUÇÃO}

Os primeiros casos da Síndrome da Imunodeficiência Adquirida-AIDS surgiram no ano de 1981, nos Estados Unidos. A síndrome até então desconhecida atingia o sistema imunológico, que inicialmente suspeitou-se ser incidente

Correspondente/Corresponding: ${ }^{*}$ Carla Jaqueline Silva Sampaio-End.: Laboratório de Microbiologia Aplicada e Bioprospecção, Instituto de Ciências da Saúde, Departamento de Biointeração, Universidade Federal da Bahia, Campus Canela, CEP: 40110-100, Salvador, Ba-E-mail: sampaiosjcarla@gmail.com - Tel: apenas em homens homossexuais e bissexuais, levantando a hipótese de que algum aspecto no estilo de vida homossexual era a provável causa para a deficiência imunológica (BRITO; CASTILHO; SZWARCWALD, 2005). No Brasil, no ano de 1982, foi identificado um jovem homossexual na cidade de São Paulo, que havia viajado anos antes para os Estados Unidos, sendo este o primeiro paciente brasileiro do qual se tem conhecimento com a soropositividade para o vírus HIV. No final da década de 80 , a frequência de casos de AIDS entre mulheres aumentou consideravelmente quando 
a transmissão heterossexual passou a ser a principal via de disseminação (MAIA; GUILHEM; FREITAS, 2008).

Complementando essa evolução, à medida que o complexo da doença foi se tornando mais amplamente conhecido e reconhecido, foi possível associar a AIDS a outros meios de transmissão, desvinculando da exclusividade da homossexualidade. As mesmas infeç̧ões e tumores foram diagnosticados em usuários de drogas injetáveis, hemofílicos do tipo A, cônjuges, parceiros sexuais e filhos de pessoas com AIDS, além de receptores de sangue ou componentes sanguíneos de indivíduos infectados com AIDS. Logo, evidenciou-se e que a AIDS era transmitida através de uma troca de fluídos corporais, em especial sangue e sêmen (MOURA; FARIA, 2017).

No que tange ao agente etiológico da doença, o vírus HIV pertence à família dos retrovírus, e é classificado na subfamília dos Lentiviridae. Ao penetrar no corpo humano, o vírus infecta, sobretudo, os linfócitos T CD4+, ligando-se ao receptor $\mathrm{CD} 4+$, em seguida alterando o DNA da célula e elaborando cópias de si mesmo. Após a multiplicação, o vírus rompe a membrana da célula promovendo a morte celular, e prossegue em busca de outros linfócitos como meio de proliferar a infecção. Frente essa realidade, o sistema imunológico perde gradativamente a capacidade de reagir às infecções exógenas e endógenas, tornando o corpo vulnerável à doenças oportunistas (ROBBINS; COTRAN; KUMAR, 2000).

Dentro dessa temática, artigos científicos sobre os casos de AIDS na cidade de Alagoinhas - BA e microrregião foram avaliados na Biblioteca Virtual em Saúde (BVS), durante o período de 2007 a 2017. Como resultado preliminar, evidenciou-se o fato de haver uma lacuna cientifica relacionada à existência de dados a respeito das características epidemiológicas e demográficas dos pacientes infectados pelo HIV e acometidos pela AIDS, visto que não foram encontrados artigos especificamente sobre a cidade de Alagoinhas e região de saúde.

Considerando que a cidade de Alagoinhas é um município com aproximadamente 160 mil habitantes (IBGE, 2018), que possui 160 anos de emancipação político-administrativa, sendo uma das mais novas cidades da Bahia, foram levantadas questões acerca da saúde pública.

Em adição, Alagoinhas caracteriza-se por ser o segundo maior polo comercial do interior do estado, visto que possui matéria-prima de qualidade e em abundância, sendo alvo das principais indústrias de cerveja, refrigerante e água do país (BRASIL, 2017). Diante desse cenário econômico, fazem-se necessários estudos que evidenciem a situação epidemiológica quanto à disseminação do vírus HIV na cidade de Alagoinhas e microrregião de saúde, que é constituída por 17 municípios (Acajutiba, Alagoinhas, Aporá, Araças, Aramari, Cardeal da Silva, Catu, Crisópolis, Entre Rios, Esplanada, Inhambupe, Itanagra, Itapicuru, Ouriçangas, Pedrão, Rio Real e Sátiro Dias).

Dessa forma, faz-se necessário analisar o perfil sociodemográfico e epidemiológico dos pacientes com HIV/AIDS com o intuito de promover o arcabouço teórico essencial à realização de melhorias estruturais no sistema de saúde da cidade e conscientizar a população para os riscos e cuidados necessários advindos dessa enfermidade.

\section{METODOLOGIA}

O presente estudo trata-se do levantamento de dados acerca dos casos notificados de AIDS na cidade de Alagoinhas e microrregião de saúde. Realizou-se pesquisa qualitativa nas bases de dados Scielo, PUBMED e Medline no período de 2007 - 2017, utilizando como estratégia de busca as palavras-chave "HIV", "AIDS" e "Alagoinhas" e operadores booleanos OR e AND para ampliar o número de artigos relacionados ao tema. Os critérios de inclusão utilizados para selecionar os artigos foram: ser artigo original; o estudo ser realizado em humanos; estar nas línguas portuguesa e inglesa; ter avaliado o quantitativo de casos registrados. Foram incluídos artigos publicados a partir do ano de 2007, com o intuito de avaliar os artigos mais recentes sobre essa temática.

Os dados secundários foram coletados através da base de dados do Sistema de Informação de Agravos e Notificação - SINAN, utilizando as variáveis: escolaridade, faixa etária, categoria de exposição, relação sexual, evolução e raça, disponibilizados pelo departamento de informática do Sistema Único de Saúde (DATASUS), e analisados empregando o programa Microsoft Excel 2010.

\section{RESULTADOS}

Durante o período 2007 - 2017, constatou-se um quantitativo de 459 casos registrados no SINAN de AIDS em adultos na Região de Saúde do município de Alagoinhas, Bahia - Brasil. Os dados foram extraídos do SINAN pautados em sete variáveis, que foram divididas em duas categorias: variáveis epidemiológicas e variáveis sóciodemográficas.

A Figura 1 exibe o número de casos de AIDS em adultos entre 15 e 79 anos, durante o período 2007-2017. Observa-se uma tendência de crescimento no número de casos na microrregião de Alagoinhas, acentuado no ano de 2014 (95 casos), e com considerável aumento constatado no ano de 2017 (90 casos).

Figura 1 - Número de casos de AIDS em adultos na Região de Saúde de Alagoinhas - BA, durante o período 2007-2017.

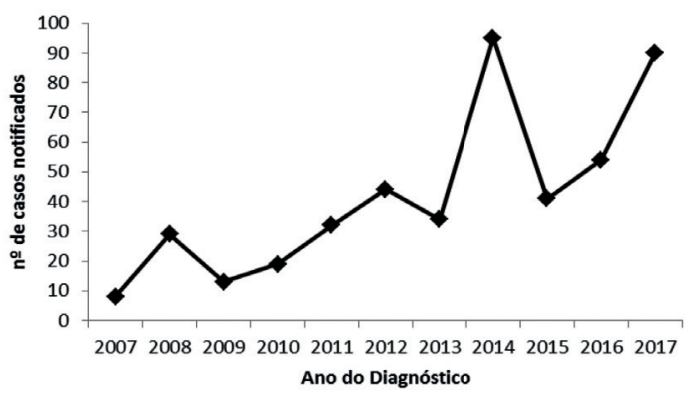

Fonte: SESAB/SUVISA/DIVEP/SINAN - Sistema de Informação de Agravos de Notificação 
Os dados da Figura 1 corroboram com as taxas de incidência durante os anos de 2007 a 2017 referentes ao município de Alagoinhas (Tabela 1), o maior é número de casos registrados (total de 211 casos), em que se observa picos de incidência da doença nos anos de $2014(36,47)$ e $2017(23,72)$. As taxas de prevalência também encontram-se elevadas, com tendência crescente no período de tempo analisado.

Tabela 1 - Taxas de incidência e prevalência de AIDS em adultos (por 100 mil hab.), no município de Alagoinhas, Bahia, Brasil, durante os anos de 2007-2017.

\begin{tabular}{lccccccccccc}
\hline & $\mathbf{2 0 0 7}$ & $\mathbf{2 0 0 8}$ & $\mathbf{2 0 0 9}$ & $\mathbf{2 0 1 0}$ & $\mathbf{2 0 1 1}$ & $\mathbf{2 0 1 2}$ & $\mathbf{2 0 1 3}$ & $\mathbf{2 0 1 4}$ & $\mathbf{2 0 1 5}$ & $\mathbf{2 0 1 6}$ & $\mathbf{2 0 1 7}$ \\
\hline Incidência & 1,51 & 13,85 & 5,08 & 6,34 & 4,90 & 16,73 & 11,14 & 36,47 & 6,47 & 14,80 & 23,72 \\
Prevalência & 5,27 & 14,58 & 18,87 & 11,27 & 10,50 & 20,91 & 23,60 & 42,98 & 41,43 & 20,60 & 37,18 \\
\hline
\end{tabular}

Fonte: IBGE/SESAB/SUVISA/DIVEP/SINAN - Sistema de Informação de Agravos de Notificação

Dentre os municípios que compõem a microrregião de saúde, a distribuição dos casos notificados de AIDS na região de saúde de Alagoinhas totaliza 459 (Figura 2), observando-se maior concentração de casos no município de Alagoinhas (45,7\%), seguido de Entre Rios $(9,4 \%)$ e Catu (9\%).

Figura 2 - Número de casos notificados de AIDS em adultos por municípios que compõem a região de saúde do município de Alagoinhas-BA, Brasil, 2007-2017.

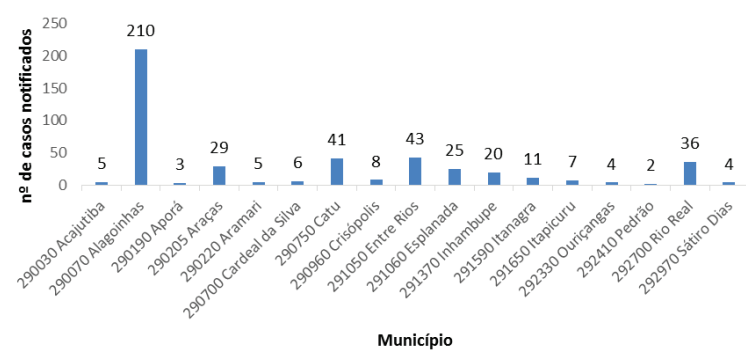

Fonte: SESAB/SUVISA/DIVEP/SINAN - Sistema de Informação de Agravos de Notificação

Ao avaliar os casos notificados de AIDS segundo as variáveis faixa etária e sexo (Figura 3), evidenciou-se que dos 459 casos notificados, 227 pertenciam ao sexo masculino e 232 pertenciam ao sexo feminino, havendo predomínio de casos, todavia, na faixa etária entre 20 a 34 anos. Dos indivíduos pertencentes a essa faixa etária, 98 $(43,17 \%)$ eram do sexo masculino e $91(39,22 \%)$ eram do sexo feminino. Nas demais faixas etárias e sexo, os casos de indivíduos do sexo feminino é superior.

Figura 3 - Número de casos notificados de AIDS em adultos segundo sexo e faixa etária, Região de Saúde do município de Alagoinhas-BA, Brasil, 2007-2017.

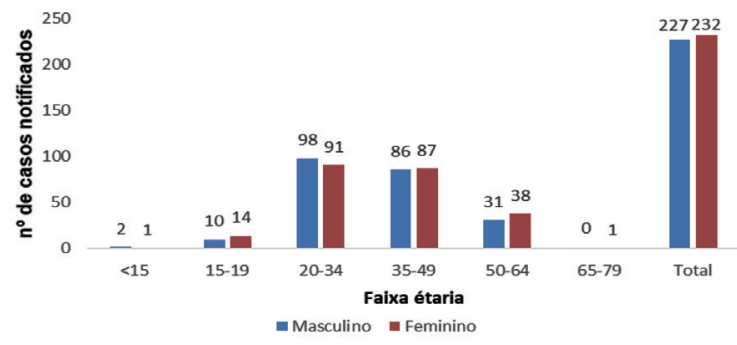

Fonte: SESAB/SUVISA/DIVEP/SINAN - Sistema de Informação de Agravos de Notificação
Verificou-se que os indivíduos com menor escolaridade foram mais acometidos, com um quantitativo de 52 pessoas com 5 a a 8 séries incompletos, 30 pessoas com 1a a 4a série incompletos e 06 pessoas analfabetos (Figura 4).

Figura 4 - Número de casos notificados de AIDS em adultos por escolaridade, Região de Saúde do município de Alagoinhas-BA, Brasil, 2007-2017.

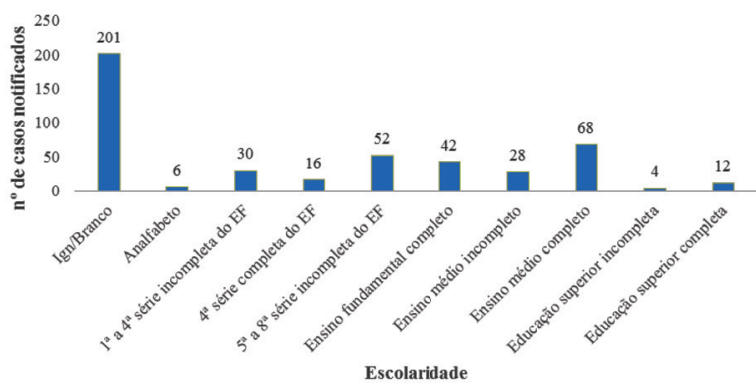

Fonte: SESAB/SUVISA/DIVEP/SINAN - Sistema de Informação de Agravos de Notificação

Quando analisado o número de casos segundo faixa etária e categoria de exposição, a maioria foi em adultos, faixa etária de 20-34 e 35-49, e dentre estes, o número de casos foi maior em indivíduos que têm relação heterossexual, seguido de indivíduos que têm relação homossexual (Tabela 2).

A faixa etária de 50-64 apresentou o total de 69 casos notificados, com maior predominância em pessoas que têm relações heterossexuais (53). A faixa etária de 15-19 teve um total de 24 casos notificados e evidenciou-se elevação no número de pessoas heterossexuais acometidas (15). Na faixa etária de $<15$ houve um total de 02 casos notificados, sendo um de relação heterossexual e um de via perinatal. A faixa etária de 65-79 é a menos afetada, apresentando apenas 01 caso, sendo esse proveniente de relação heterossexual. 
Tabela 2 - Número de casos notificados de AIDS em adultos segundo faixa etária e categoria de exposição, Região de saúde do município de Alagoinhas-BA, Brasil, 2007-2017.

\begin{tabular}{|c|c|c|c|c|c|c|c|}
\hline Faixa Etária SINAN & Ignorado & Homossexual & Bissexual & Heterossexual & $\begin{array}{l}\text { UDI (Uso de } \\
\text { Drogas Injetáveis) }\end{array}$ & Perinatal & Total \\
\hline$<15$ & - & 1 & - & 1 & - & 1 & 3 \\
\hline $15-19$ & 3 & 3 & 1 & 15 & 1 & 1 & 24 \\
\hline $20-34$ & 18 & 31 & 5 & 122 & 9 & 4 & 189 \\
\hline $35-49$ & 18 & 15 & 3 & 129 & 4 & 4 & 173 \\
\hline $50-64$ & 8 & 5 & 1 & 53 & 1 & 1 & 69 \\
\hline $65-79$ & - & - & - & 1 & - & - & 1 \\
\hline Total & 47 & 55 & 10 & 321 & 15 & 11 & 459 \\
\hline
\end{tabular}

Fonte: SESAB/SUVISA/DIVEP/SINAN - Sistema de Informação de Agravos de Notificação

A partir da análise do número total de casos de todas as faixas etárias, fica evidente o número aumentado de pessoas que possuem relação heterossexual (321), seguido de pessoas com relação homossexual (55), ignorado (47), UDI (11), relação bissexual (10) e via perinatal (11). Os resultados da pesquisa mostram que a principal via de transmissão foi a sexual, sendo maior número de casos por via heterossexual, corroborado pelo estudo de Santos e Zambenedetti (2016).

Quando analisado o número de casos notificados de AIDS por ralação sexual foi perceptível o alto número de casos em indivíduos que possuem relações sexuais só com homens, totalizando 258 , seguido de só com mulheres (135) ignorado/ branco (52) (Figura 5).

Figura 5 - Número de casos notificados de AIDS em adultos por relação sexual, Região de saúde do município de AlagoinhasBA-Brasil, 2007-2017.

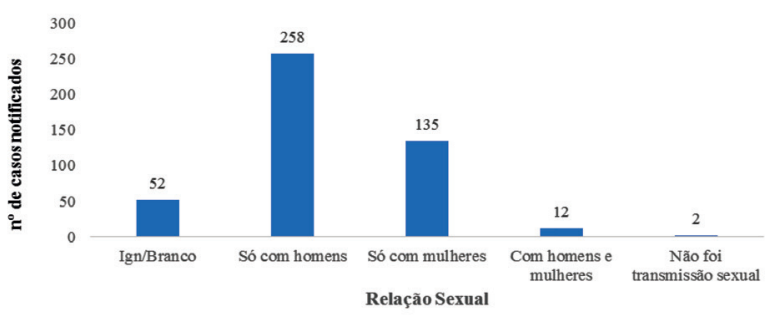

Fonte: SESAB/SUVISA/DIVEP/SINAN - Sistema de Informação de Agravos de Notificação

No que tange a evolução da doença, foram registrados um total de 33 óbitos por AIDS na região de saúde do munícipio de Alagoinhas-BA durante o período de 2007 a 2017, evidenciando, assim, a persistência de mortes mesmo após a introdução de antirretrovirais como tratamento para a doença (Figura 6).
Figura 6 - Casos notificados de AIDS em adultos segundo evolução, Região de saúde do município de Alagoinhas-BA, Brasil, 2007-2017.

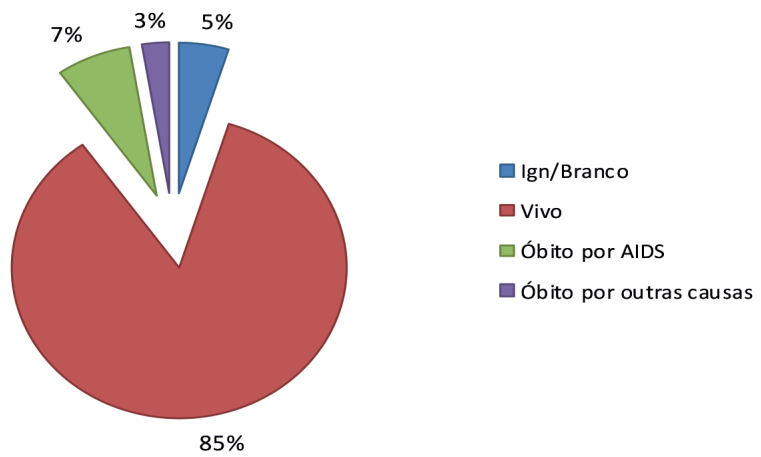

Fonte: SESAB/SUVISA/DIVEP/SINAN - Sistema de Informação de Agravos de Notificação

A análise do número de casos notificados de AIDS, segundo raça e ano de notificação, evidencia o maior número de casos no ano de 2014, sendo que Ignorados/ Brancos possuíram maior quantidade de notificações (53) neste ano (Figura 7).

Figura 7 - Número de casos notificados de AIDS segundo raça, Região de saúde do município de Alagoinhas-BA, 2007-2017.

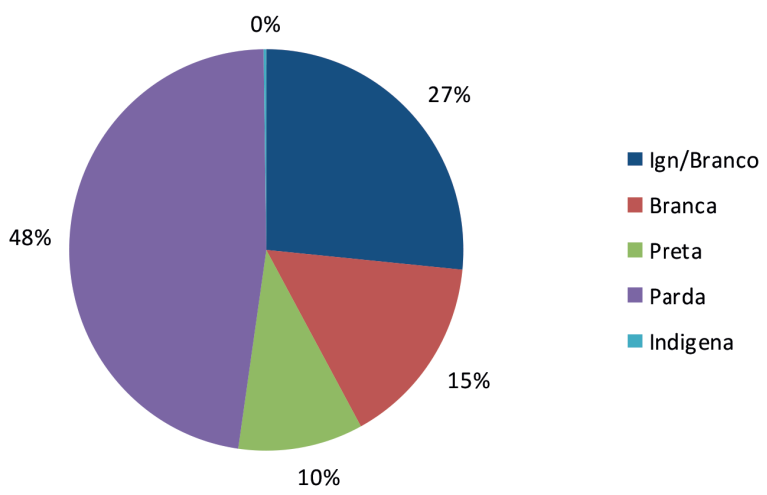

Fonte: SESAB/SUVISA/DIVEP/SINAN - Sistema de Informação de Agravos de Notificação 
Durante o período estudado, a raça que obteve maior percentual de notificações foi a parda $48 \%$ (218 casos), seguido da raça branca $15 \%$ (71) e preta $10 \%$ (47).

\section{DISCUSSÃO}

O aumento no número de casos de adultos com HIV/ AIDS observado reflete o panorama para a região Nordeste, que retrata o número crescente na taxa de detecção da doença durante o período de 2006-2016, sobretudo, após o ano de 2012, segundo o Boletim Epidemiológico HIV/AIDS (BRASIL, 2017). Devido à determinação do Ministério da Saúde (PORTARIA № 1.271, de 6 de Junho de 2014), que incluiu a infecção por HIV na Lista Nacional de Notificação Compulsória de Doenças, Agravos e Eventos de Saúde Pública, a partir do ano de 2014, atribui-se, portanto, o aumento considerável nos casos de indivíduos que desenvolveram a AIDS após o ano de 2014 à notificação regular realizada pelos profissionais de saúde.

Existe semelhança na distribuição proporcional dos casos nos municípios de Entre Rios e Catu. Porém, Alagoinhas também é o município com maior número de casos proporcionalmente comparados com sua população estimada em 150.832 segundo IBGE para o ano de 2018 com $0,14 \%$ das notificações, em consonância com os elevados índices de incidência e prevalência da doença. O município possui um CTA (Centro de Testagem e Aconselhamento) que funciona de forma ativa e coordenada com a Secretaria de Saúde, sob a forma de divulgação dos serviços prestados para a população local.

O último Boletim Epidemiológico AIDS-DST 2017 indicou um percentual de $52,5 \%$ dos casos de AIDS em indivíduos na faixa etária de 20 a 34 anos, durante o período de 2007 a 2017. Segundo Idele et al. (2014), são necessárias várias intervenções para a redução da vulnerabilidade à infecção pelo HIV entre adolescentes, assim como adultos, com destaque para os compromissos políticos e políticas que tratam das questões de pobreza, baixos níveis de educação, marginalização de particular grupos populacionais, estigma e discriminação, associados aos grupos ou comportamentos específicos, entre outros. Ainda de acordo com os autores, a política e os esforços do programa em todos os setores de desenvolvimento, juntamente com o ambiente favorável, são fatores que têm influência significativa sobre a eficácia e o sucesso das intervenções que reforcem o conhecimento do HIV e melhorias no acesso aos testes, cuidados e serviços de tratamento.

Pessoas com faixa etária mais elevada começam a surgir no cenário dos indivíduos que vivem com AIDS e, diante das condições sociodemográficas e econômicas desfavoráveis em que grande parte se encontra, este fato deve ser visto como um grande problema de saúde pública a ser enfrentado (OKUNO et al., 2014). O estudo dos autores revelou que idade, renda e tempo de diagnóstico foram as variáveis que mais responderam pela alteração da qualidade de vida dos idosos que vivem com AIDS.
Neste sentido, Silva et al. (2018) corrobora com os dados obtidos no presente estudo. Pois, segundo os autores, os casos de AIDS em pessoas acima de 50 anos ( 60 a 69 anos) também vêm aumentando, fato relevante para as equipes de saúde, devido ao impacto que a doença exerce nesses indivíduos, revestindo-se de grande importância epidemiológica. Os autores também ressaltam a importância do desenvolvimento de ações que promovam a saúde do idoso, sobretudo no que tange a apropriação da doença e medidas de prevenção.

Os dados evidenciam que a falta de instrução é relevante na infecção pelo vírus HIV no que tange a microrregião de saúde a qual está inserida o município de Alagoinhas-BA. Esses dados são confirmados pelo Boletim Epidemiológico de HIV/AIDS (2017) que também retrata predomínio de pessoas com baixo nível de escolaridade dentre os pacientes vivendo com AIDS no Brasil.

Diferenças de acesso à informação, perspectivas e possibilidades são reflexos do grau de escolaridade de uma população. Este é um indicador de análise de dados sóciodemográficos, comumente associado a níveis de renda, e um indicador indireto da situação socioeconômica de uma população. No entanto, apesar de possuírem acesso à informação sobre a doença e os mecanismos de prevenção, o conhecimento aprofundado sobre o tema ainda é deficitário (SILVA et al., 2016).

A epidemia no Brasil iniciou-se em grupos com elevado grau de informação e no decorrer do tempo se disseminou para pacientes com baixos níveis de escolaridade. $O$ grau de instrução constitui um parâmetro do nível socioeconômico da população, de tal maneira que o aumento da incidência de AIDS entre indivíduos com baixo grau de escolaridade tem caracterizado a pauperização da epidemia no Brasil (MELO et al., 2019).

Adicionalmente, neste estudo, a maioria dos pacientes apresenta o 1 o grau completo, estando em consonância com o grau de escolaridade da população de Alagoinhas no censo de 2010 em que $55,47 \%$ dos entrevistados possuíam o 2 - ciclo fundamental completo ou escolaridade superior (IBGE, 2018). Em contrapartida, no estudo de Toledo et al. (2010), realizado no estado do Espírito Santo, a maioria dos pacientes apresentava 1ㅇ grau incompleto. Essa redução do grau de instrução também ocorre quando comparado ao grau de instrução dos indivíduos durante o início da epidemia na década de 80. Ainda de acordo com os autores, a falta de conhecimento confere ao indivíduo menor acesso à informação e, consequentemente, aos serviços de assistência, o que favorece a disseminação da doença em indivíduos com menor grau de instrução.

Atualmente, a transmissão sexual possui grande relevância, pois, segundo Feitosa et al. (2018) além de homossexuais, os heterossexuais têm contribuído para o aumento da incidência da transmissão, devido à crença de que sua orientação sexual traz segurança/proteção contra a doença, negligenciando o uso de preservativo. Fato que leva os heterossexuais a ter vários parceiros, ocorrendo, 
também, elevação do índice de mulheres contaminadas. Em adição, os autores relatam que a AIDS é descoberta por acaso entre os heterossexuais, pois estes associam a doença à homossexualidade.

Com a expansão da AIDS entre heterossexuais passou-se a exprimir sobre comportamento de risco e vulnerabilidade (REIS; GIR, 2009; MAIA et al., 2019). Ao analisar os dados colhidos e os estudos realizados, tornou-se possível traçar um paralelo entre a realidade da região de saúde e a realidade descrita pelos autores. Através deste, observou-se que os estudos definem a situação atual da região, à medida que houve um número significativo de casos onde a transmissão foi sexual, levando em consideração as relações sexuais só com homens, só com mulheres, com homens e mulheres.

O abandono do tratamento é um fator relevante e que implica no número de óbitos. De acordo com Rodrigues e Maksud (2017), o Ministério da Saúde possuía no ano de 2009 dois critérios que sugeriam o abandono de tratamento: pacientes que ao retirar os seus medicamentos não retornaram ao serviço em três meses e pacientes que não se apresentam à consulta em um período de 6 meses, o que favorece o aumento do número de óbito.

Embora seja observada uma diminuição nas hospitalizações e óbitos por AIDS, a procura tardia por auxílio médico resulta na persistência de casos diagnosticados somente após doenças oportunistas instaladas, interferindo, assim, na boa progressão do tratamento e aumentando o risco de disseminação do vírus (ROSSI et al., 2012). Desta forma, os óbitos notificados na região de saúde de Alagoinhas 7\% (33 óbitos) podem estar relacionados ao diagnóstico tardio, administração incorreta do medicamento e abandono do tratamento, resultando na redução da taxa de sobrevida e consequente morte.

O aumento no número de casos de AIDS em pardos e brancos é atribuído a uma maior distribuição dessas raças em relação às demais. Nesse sentido, é necessário considerar os diferentes grupos raciais, levando em conta a alta miscigenação do país, em busca de criar ou aprimorar programas de prevenção para os grupos mais acometidos. Destacando a necessidade de capacitação dos profissionais de saúde para melhorias no acolhimento, em busca de otimização e confiabilidade na atenção básica (PEDROSA et al., 2015).

\section{CONCLUSÃO}

O presente estudo permitiu compreender que os indivíduos adultos vivendo com a AIDS, no período de 2007 a 2017 possuem o seguinte perfil: predominando no sexo feminino, na faixa etária de 20-34 anos, com baixo nível de escolaridade (5a a 8a série incompletos).

Na região de saúde analisada, a AIDS prevalece em pessoas que têm relações heterossexuais, na faixa etária de 50-64 anos, e, por relação sexual predomina relações só com homens, sendo a aquisição da infecção, sobretudo, na raça parda. Dessa forma, nota-se a importância de campanhas e orientações, com o objetivo de prevenção, além de uma equipe multiprofissional, para que haja a promoção de assistência integral ao paciente. A melhoria do acesso aos serviços, qualidade da atenção, pluralidade do cuidado e direcionamento aos grupos vulneráveis proporciona uma maior qualidade de vida aos pacientes.

\section{REFERÊNCIAS}

BRASIL, Ministério da Saúde. Secretaria de Vigilância em Saúde. Boletim Epidemiológico de HIV/AIDS, Brasil, 2017.

BRITO, A. M.; CASTILHO, E. A.; SZWARCWALD, C. L. Regional patterns of the temporal evolution of the AIDS epidemic in Brazil following the introduction of antiretroviral therapy. Braz. J. Infec. Dis., Salvador, v. 9, n. 1, p. 9-19, 2005.

FEITOSA, P. W. G. et al. De "Peste Gay" à Supremacia da AIDS entre Heterossexuais no Brasil. Rev. Mult. Psic., [s.], v. 12, n. 42, p. 651-661, 2018.

IDELE, P. et al. Epidemiology of HIV and AIDS among adolescents: current status, inequities, and data gaps. J. Acquir Immune Defic. Syndr., Hagerstown, v. 66, p. S144-S153, 2014.

INSTITUTO BRASILEIRO DE GEOGRAFIA E ESTATÍSTICA (IBGE). 2018. Disponível em: https://ibge.gov.br/ Acesso em: 20 ago. 2018.

MAIA, C.; GUILHEM, D.; FREITAS, D. Vulnerabilidade ao HIV/Aids de pessoas heterossexuais casadas ou em união estável. Rev. Saúde Pública, São Paulo, v. 42, p. 242-248, 2008.

MAIA, D. A. C. et al. Notificação de casos de HIV/AIDS em adolescentes portadores de HIV/Aids no Nordeste: série histórica entre os anos de 2004 a 2014. Revista Diálogos Acadêmicos, Fortaleza, v. 7, n. 1, 2019.

MELO, M. C. et al. Sobrevida de pacientes com aids e associação com escolaridade e raça/cor da pele no Sul e Sudeste do Brasil: estudo de coorte, 1998-1999. Epidemiol. serv. saude., Brasília, v. 28, 2019.

MOURA, J. P.; FARIA, M. R. Caracterização e perfil epidemiológico das pessoas que vivem com HIV/AIDS. Rev. enferm. UFPE on line, Recife, $v$. 11, n. supl. 12, p. 5214-5220, 2017.

OKUNO, M. F. P. et al. Qualidade de vida de pacientes idosos vivendo com HIV/AIDS. Cad. Saúde Pública, Rio de Janeiro, v. 30, p. 1551-1559, 2014.

PEDROSA, N. L. et al. The historic data series on AIDS in the state of Ceará, Brazil. Ciênc. Saúde Colet., Rio de Janeiro, v. 20, n. 4, p. 1177-1184, 2015.

ROBBINS, S.L.; COTRAN, R.S.; KUMAR, V. Fundamentos de Robbins patologia estrutural e funcional. 6. ed. Rio de Janeiro: Guanabara Koogan, 2000. 766 p.

REIS, R. K.; GIR, E. Vulnerabilidade ao HIV/AIDS e a prevenção da transmissão sexual entre casais sorodiscordantes. Rev. Esc Enferm USP, São Paulo, v. 43, n. 3, p. 662-669, 2009.

RODRIGUES, M.; MAKSUD, I. Abandono de tratamento: itinerários terapêuticos de pacientes com HIV/Aids. Saúde Debate, Londrina, v. 41, p. 526-538, 2017

ROSSI, S. M. G. et al. Impacto da terapia antirretroviral conforme diferentes consensos de tratamento da Aids no Brasil. Rev. Panam Salud Pública, Washington, v. 32, p. 117-123, 2012.

SANTOS, E. A. P.; ZAMBENEDETTI, G. Caracterização dos casos de HIV/ AIDS no município de Irati, PR, no período de 1994 a 2012. Rev. Psicol. Saúde, [s.I], v. 8, n .2, p. $97-112,2016$.

SILVA, B. N. da et al. Panorama epidemiológico da AIDS em idosos. 
Hygeia, Revista Brasileira de Geografia Médica e da Saúde, Uberlândia, v. 14, n. 29, p. $80-88,2018$.

SILVA, I. R. et al. Orders and disorders: the complexity of adolescence and sexual health - -contributions to nursing. Rev. Enferm. UERJ, Rio de Janeiro, v. 24, n. 2, 2016.

TOLEDO, L. S. G. et al. Características e tendência da AIDS entre idosos no Estado do Espírito Santo. Rev. Soc. Bras. Med. Trop., Brasília, v. 43, n. 3, 2010.

UNAIDS Brasil. A ONU e a resposta à AIDS no Brasil. 3. ed. Brasília: Unaids Brasil, 2013. Disponível em: http://www.unaids.org.br/. Acesso em: 18 nov. 2018.

Submetido em: 29/05/2019

Aceito em: 18/11/2019 